\title{
Diseño y construcción de reflectores solares en pétalos: paraboloide y catenoide
}

\author{
Design and construction of petal solar reflectors: paraboloid and catenoid
}

\author{
César Medina*10, Sandra Velazco $\sqrt{10}$ \\ ${ }^{1}$ Universidad Nacional de Tucumán, Facultad de Ciencias Exactas y Tecnología, Tucumán, Argentina.
}

Recibido en 01 de Enero, 2020. Revisado en 10 de Abril, 2020. Aceptado en 23 de Mayo, 2020.

\begin{abstract}
Se presenta un estudio geométrico detallado de distintos diseños de reflectores solares basados en la metodología de "diagramas de pétalos". Se analiza el modelo paraboloide y se introduce un nuevo modelo, el catenoide, discutiendo comparativamente sus ventajas y desventajas. Se presentan diseños convencionales y optimizados de ambos tipos de superficie, analizando cuantitativamente diversos aspectos críticos tales como la distancia focal, el área efectiva y la cantidad de pétalos. Se ofrecen criterios y sugerencias para seleccionar los materiales y planificar el tamaño del sistema según su finalidad. Se presentan algunos modelos construidos que evidencian una buena convergencia de los rayos reflejados.
\end{abstract}

Palabras clave: Reflectores solares, Diagrama de pétalos, Paraboloide, Catenoide.

A detailed geometric study is presented of different designs of solar reflectors based on the methodology of petal diagrams. The paraboloid model is analyzed and a new model is introduced, the catenoid, discussing its advantages and disadvantages on a comparative basis. Conventional and optimized designs are presented for both kinds of surfaces, analyzing quantitatively several critical aspects such as focal distance, effective area and number of petals. Criterions and suggestions are offered to select materials and to plan the size of the system according to its purpose. Some constructed models are presented which evince a good convergence of reflected rays.

Keywords: Solar reflectors, Petal diagrams, Paraboloid, Catenoid.

\section{Introducción}

En la actualidad, existe un acuerdo muy extendido, entre los especialistas en óptica, respecto de la idoneidad del modelo de la óptica geométrica a los fines de estudiar los fenómenos de refracción y reflexión; en particular, en sistemas construidos por el hombre, tales como lentes y espejos, siempre que el grado de pulimento de las superficies de los mismos permita suponer que sus irregularidades son mucho menores que las longitudes de onda del espectro visible, y que el tamaño de los sistemas (lentes o espejos) sea bastante grande como para despreciar efectos de difracción.

Este amplio consenso reafirma un vínculo evolutivo, entre la geometría y la óptica, que se remonta a la antigüedad clásica. La Catróptica, uno de los primeros tratados sobre la teoría de los espejos, se atribuye a Euclides (325-265 a.C.), conocido como el "padre de la geometría" por haber presentado las propiedades fundamentales de las formas regulares en su célebre obra Elementos [1, 2]. El diseño, construcción y aplicación práctica de los reflectores solares también data de hace muchos siglos. Entre las tradiciones más conocidas se encuentra la de los "espejos ustorios" de Arquímedes, que supuestamen-

*Correo electrónico: cfmedina@herrera.unt.edu.ar te incendiaron los barcos romanos durante el sitio de Siracusa (214-212 a.C.) [3]. Esta hazaña bélica no ha sido confirmada por historiadores reconocidos y es difícil saber cuánto hay en ella de historia o leyenda, pero técnicamente es factible, aun con los limitados recursos de entonces ${ }^{1}$ Desde aquellas épocas remotas hasta la actualidad, la concentración de energía solar ha sido y continúa siendo una de las metodologías más usadas para obtener altas temperaturas en forma rápida, relativamente sencilla, económica, y sin efectos nocivos sobre el ambiente.

Los reflectores paraboloides se han usado en iluminación desde hace mucho tiempo, pero las propiedades ventajosas de esta forma geométrica se han popularizado en épocas recientes gracias a la divulgación de ciertas tecnologías de comunicación (radares, antenas satelitales, etc.) y del uso de las cocinas solares. Actualmente existen muchos sitios de internet donde se ponderan las ventajas de estos artefactos y se ofrece material técnico -por lo general de poca precisión- tales como planos,

\footnotetext{
${ }^{1}$ Una de las estrategias romanas consistía en asaltar las fortalezas con escaleras accionadas por poleas desde los barcos, para lo cual éstos deben haber atracado muy cerca de las murallas y mantenerse quietos. Además, debe tenerse en cuenta que los barcos antiguos se construían con materiales fácilmente inflamables (velamen de tela y casco de madera).
} 
videos, etc., para construir reflectores solares más o menos sencillos y de bajo costo. Este tipo de material tiene una considerable demanda por parte de aficionados al bricolaje y a la vida al aire libre, al tiempo que es promocionado por distintos grupos ecologistas. De hecho, la energía solar es un importante tema de investigación a nivel mundial, en especial en países donde las condiciones geográficas y climáticas son propicias para su aprovechamiento, y constituye una alternativa valiosa en zonas donde el combustible y las instalaciones eléctricas son escasos o costosos $[4,5,6,7,8,9]$.

Aparte de estas razones, creemos que el interés que suscita este tema en muchas personas se debe a otras cuestiones. En nuestra experiencia docente, hemos comprobado que los experimentos de óptica entusiasman a los estudiantes, en gran medida, por su estética visual: brillo, colores, etc. Pero en el caso de los reflectores solares existe algo que les resulta más asombroso y fascinante: el hecho de descubrir, o constatar, que un fenómeno tan común y cotidiano como la radiación solar ofrece la posibilidad de captar, dirigir y concentrar una gran cantidad de energía, mediante un recurso muy simple y accesible. Este simple hecho puede motivar al estudiante a profundizar su conocimiento y comprensión del fenómeno, y a incrementar su capacidad de manipularlo con fines específicos.

Los detalles matemáticos expuestos en este trabajo tienen por objeto ofrecer una base técnica para que el lector pueda elegir el diseño más apropiado a sus fines, que ya sean didácticos o prácticos, exigirán una dada precisión. Desde luego, en el aula o laboratorio, el docente deberá adaptar, resumir o simplificar los contenidos de acuerdo al nivel curricular del curso.

En cuanto a la construcción, creemos conveniente plantearla como un proyecto experimental de la clase, dividiendo a los estudiantes en pequeños grupos que podrían armar diferentes diseños, con fines comparativos. Esta etapa también requiere de cierta prolijidad y precisión que sólo se adquiere con la experiencia, y por ello el docente deberá ofrecer una guía muy atenta; pero a los fines de un aprendizaje significativo, es más importante la participación activa de los estudiantes que la precisión del resultado final [10].

Desde el punto de vista didáctico, la riqueza del tema permite conectar contenidos de óptica geométrica, matemática y física solar en un enfoque unificado que concuerda con las preceptivas pedagógicas modernas. Además, brinda la oportunidad de concientizar a los estudiantes en el uso responsable de los recursos y la conveniencia de emplear energías económicas y no contaminantes.

\section{Primera Parte: El Reflector Parabólico}

\subsection{Fundamentos geométricos}

El paraboloide de revolución, es decir, de sección transversal circular, es la única superficie que, al menos ideal- mente, puede reflejar en un solo punto - su foco- un haz de rayos que incidan paralelos a su eje. Por tal motivo es el concentrador, por excelencia, de energía solar. Para que los rayos solares incidan paralelos al eje del paraboloide, éste puede orientarse hacia el sol con relativa facilidad, sobre todo en los diseños livianos como los que se estudian en este trabajo. La orientación puede efectuarse manualmente, en forma aproximada, o bien por medios automáticos más precisos (pero éstos no son sencillos ni de bajo costo).

Si bien un paraboloide de revolución puede construirse en una sola pieza, por ejemplo, mediante procesos de moldeado y fundición, ello supone una considerable complejidad técnica y costo instrumental. Una alternativa mucho más simple y barata es construir el llamado "paraboloide en pétalos". Para ello basta recortar una lámina plana flexible según un patrón de sectores iguales, los cuales, al unirse por sus lados contiguos, formarán el paraboloide. Las siguientes secciones desarrollan un análisis geométrico para planificar esta tarea.

\subsection{La parábola: curva generatriz}

El paraboloide de revolución, como su nombre lo indica, es la superficie que genera una parábola al girar alrededor de su eje (técnicamente, decimos que la parábola, curva plana en dos dimensiones, es la generatriz del paraboloide, superficie en tres dimensiones). La Fig. 1 muestra una parábola (curva gruesa roja) que al girar alrededor del eje $z$ como lo indica la flecha, genera el paraboloide representado con líneas de trazos.

La ecuación de una parábola con vértice en el origen puede escribirse

$$
z=\frac{x^{2}}{4 f}
$$

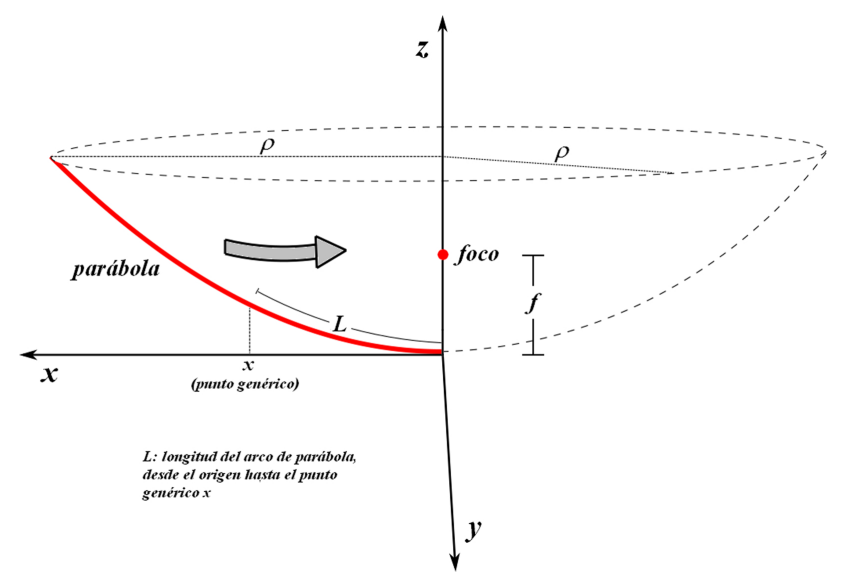

Figura 1: Parábola (curva gruesa roja) que al girar alrededor del eje $z$, genera un paraboloide (líneas de trazos). Se indica el foco (punto rojo), la distancia focal $f$, el radio $\rho$ del paraboloide, a una dada altura, y la longitud del arco de parábola $L$ desde el origen hasta un punto genérico $x$. 
donde $f$ representa la distancia focal, que se mide entre el vértice y el foco de la parábola (punto rojo en la Fig. 1).

Es claro que cuando la parábola gira alrededor del eje $z$, cualquier punto $P(x, z)$ perteneciente a ella genera una circunferencia de radio $x$, a una altura $z$. Luego, el radio $\rho$ del paraboloide, a una dada altura $z$, será igual a la abscisa de la parábola a esa dada altura (v. Fig. 1).

Otro concepto muy importante, en este estudio, es el de longitud de un arco de curva. Para cualquier curva $z=f(x)$ en el plano $x z$, la longitud de un arco entre dos puntos de abscisas $x_{1}$ y $x_{2}$ está dada por [11]

$$
L=\int_{x_{1}}^{x_{2}} d s=\int_{x_{1}}^{x_{2}} \sqrt{1+\left(\frac{d z}{d x}\right)^{2}} d x
$$

Calculando el paréntesis del último miembro a partir de la Ec. (1) e integrando, se tiene que la longitud del tramo de parábola comprendido entre el origen (vértice de la parábola) y un punto cualquiera $P(x, z)$ perteneciente a ella está dada por

$$
L=\frac{x}{2} \sqrt{1+\frac{x^{2}}{4 f^{2}}}+f \cdot \ln \left(\frac{x}{2 f}+\sqrt{1+\frac{x^{2}}{4 f^{2}}}\right)
$$

\subsection{El paraboloide en pétalos}

Una superficie de revolución puede dividirse longitudinalmente en un número arbitrario $n$ de sectores iguales, cada uno de los cuales corresponde al giro de la generatriz en un ángulo $\phi=2 \pi / n$. La Fig. 2a (en escala aproximada) muestra un paraboloide, con su eje en dirección vertical, dividido en 4 sectores iguales (coloreados alternadamente en marrón y amarillo). Si estos 4 sectores se extienden en un plano se obtiene la Fig. 2b. Este tipo de diagrama, donde los $n$ sectores de una superficie se extienden en un plano, será llamado, en este contexto, "diagrama de pétalos" y constituye la base para la construcción de los diseños aquí propuestos.

Para que el reflector funcione eficazmente como concentrador de energía solar, la forma de los pétalos debe calcularse con precisión. Los bordes exteriores de los pétalos son simplemente arcos de una circunferencia cuyo radio es el valor máximo de $L$, pues la longitud de un pétalo es la longitud de la parábola generatriz. El cálculo de los bordes laterales requiere un análisis geométrico más detallado:

En primer lugar, debe destacarse que en la Ec. (3), la variable $x$, además de representar la abscisa de la parábola generatriz, representa el radio del paraboloide a una dada altura $z$ (v. Fig. 1). Luego, para mayor claridad en los desarrollos posteriores, conviene reescribir la Ec. (3) en términos del radio $\rho$ del paraboloide:

$$
L=\frac{x}{2} \sqrt{1+\frac{\rho^{2}}{4 f^{2}}}+f \cdot \ln \left(\frac{\rho}{2 f}+\sqrt{1+\frac{\rho^{2}}{4 f^{2}}}\right)
$$

Ahora bien, el paraboloide, a una dada altura z tiene un dado radio $\rho$, al cual le corresponde una circunferencia de
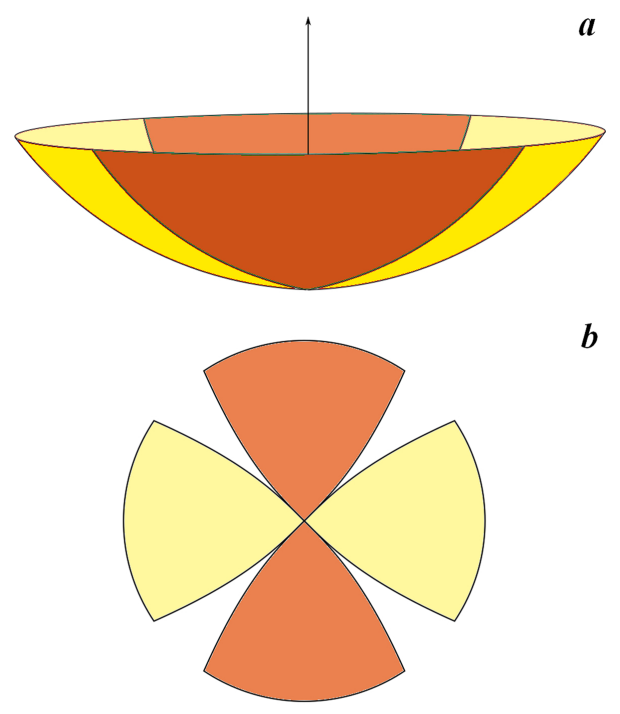

$b$

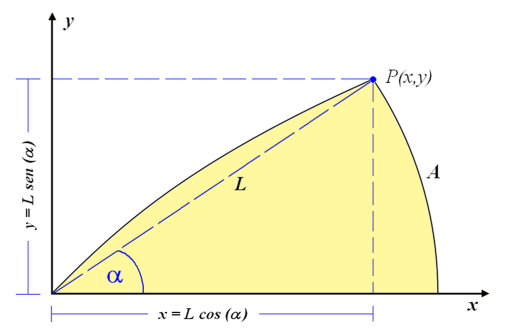

$c$

Figura 2: a) Paraboloide dividido en cuatro sectores o pétalos. b) Diagrama de pétalos. c) Mitad de un pétalo donde se indican las coordenadas de un punto genérico del borde lateral y su relación geométrica con los parámetros $L, \mathrm{~A}$ y $\alpha$.

longitud $2 \pi \rho$. Dado que el paraboloide se ha dividido en $n$ sectores iguales, a cada uno de ellos les corresponderá, a esa dada altura $z$, un arco de circunferencia de longitud $2 \pi \rho / n$. Luego, el arco de un pétalo, correspondiente a ese dado valor de $\rho$, también será $2 \pi \rho / n$, pues los pétalos son los sectores extendidos en un plano.

Dado que los pétalos son simétricos respecto de su bisectriz, a los fines de calcular los bordes laterales, basta considerar la mitad de un pétalo (Fig. 2c). A cada longitud $L$, le corresponderá un semiarco $A$ dado por

$$
A=\frac{\pi \rho}{n}
$$

Consideremos, por ejemplo, el caso del punto $P(x, y)$ de la Fig. 2c, ubicado en el vértice formado por uno de los bordes laterales y el borde externo del pétalo. Este punto subtiende al origen (centro del paraboloide) un ángulo $\alpha$ con respecto a la horizontal. Luego, las coordenadas de este punto serán (ecuaciones polares):

$$
\begin{aligned}
& x=L \cos (\alpha) \\
& y=L \operatorname{sen}(\alpha)
\end{aligned}
$$

Donde el ángulo $\alpha$ es simplemente (v. Fig 2c):

$$
\alpha=\frac{A}{L}=\frac{\pi \rho}{n L}
$$


Este mismo razonamiento se aplica a cualquier punto del borde lateral. Luego, para obtener un conjunto de coordenadas de un borde lateral, basta tomar una serie arbitraria de valores de $\rho$ entre cero y el valor del radio máximo, y reemplazar cada uno de estos valores en las Ecs. (3), (5), (6) y (7). Cabe destacar que este algoritmo es matemáticamente exacto para cualquier cantidad de pétalos $(n)$, aun cuando ésta sea muy escasa, como puede apreciarse en la Fig. 3. Esto no sólo lo hace ostensiblemente más preciso que los algoritmos de uso común entre aficionados; también es más preciso que el algoritmo propuesto en algunos trabajos científicos donde erróneamente se considera $x=L$ y $y=A$. Esto último puede ser una aproximación aceptable sólo para valores pequeños de $\alpha$, es decir, valores grandes de $n$ (v. Ecs. 5 , 6 у 7 ).

Una vez obtenido un borde lateral, el otro puede obtenerse por simetría; y una vez obtenido un pétalo, los demás pueden obtenerse por rotación. En [12] ofrecemos un programa de nuestra autoría, Programa I, desarrollado en Scilab 5.5.1, para graficar con precisión diagramas de pétalos para un paraboloide ${ }^{2}$ En este programa el usuario puede elegir el valor de la distancia focal $f$, la cantidad $n$ de pétalos, la longitud de los mismos y el tamaño en pixeles de la figura, la cual puede obtenerse directamente en formatos svg y pdf ${ }^{3}$

\subsection{Diseño eficiente de un paraboloide en pétalos}

\subsubsection{Planificación de la cantidad de pétalos}

La mayoría de los aficionados suelen construir reflectores paraboloides de muchos pétalos, pues consideran que así se aproximan más a un paraboloide de sección circular (paraboloide de revolución). Esto es cierto si se usa un
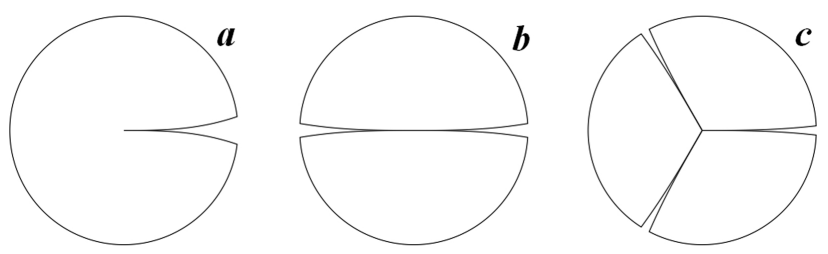

Figura 3: Diagramas de pétalos para uno, dos y tres pétalos (a, b y c, respectivamente).

\footnotetext{
2 Scilab es un software libre para análisis numérico (Licencia Cecill), con un lenguaje de programación de alto nivel para cálculo científico. Dispone de interfaces con Fortran, Java, C y $\mathrm{C}^{++}$y puede instalarse en sistemas Windows y GNU/Linux. Los paquetes de instalación pueden descargarse gratuitamente de su sitio oficial: https://www.scilab.org/

Los programas ofrecidos en este trabajo tienen la extensión convencional de Scilab: .sce. Este formato puede ser ejecutado por Scilab o bien puede ser leído y editado como texto por la aplicación "bloc de notas" de Windows, o por cualquier procesador de textos.

3 Para las longitudes se puede elegir valores numéricos arbitrarios pero éstos no indican unidades de medida sino simplemente proporciones. La escala de la figura final se dimensiona aparte, en pixeles.
}

algoritmo aproximado, como se menciona en la sección anterior; pero si se usa el algoritmo exacto propuesto en este trabajo, la importancia de la cantidad de pétalos puede tornarse secundaria frente a la de otros factores tales como la precisión en el cálculo y la técnica para recortar y unir los pétalos.

Aun así, en general no es recomendable construir paraboloides con muy pocos pétalos, y el caso extremo de un solo pétalo (Fig. 3a), aunque es matemáticamente exacto, tiene poco interés experimental, porque es prácticamente imposible construir un paraboloide de un solo pétalo sin deformaciones. Esto se debe a que la distribución de esfuerzos en la superficie será muy asimétrica, porque las zonas cercanas a la juntura (línea de unión de los lados interiores del pétalo) estarán sometidas a tensiones muy distintas de aquellas de las zonas alejadas de esta línea.

De hecho, las junturas de los pétalos, cualquiera sea su cantidad, pueden producir aristas o, al menos, discontinuidades en las pendientes de la superficie, las cuales provocan deformaciones más o menos importantes, según la rigidez del material. En láminas muy flexibles y homogéneas en toda su extensión, si los pétalos están bien calculados, recortados y acoplados, todos ellos se curvarán de un modo muy similar y la sección tenderá a ser circular, aun para una cantidad relativamente escasa de pétalos, produciendo una convergencia eficaz de los rayos reflejados.

En láminas rígidas, por lo contrario, los pétalos se curvarán muy poco, la sección tenderá a ser poligonal, no circular, y el paraboloide será un poliedro. Esto no implica necesariamente una desventaja, pues puede ser más preciso y eficaz, como concentrador, un poliedro uniforme que un paraboloide que tiende a ser circular pero tiene deformaciones importantes.

Lo que debe tenerse presente, en el caso de un poliedro de facetas planas, es que los pétalos se comportarán como un espejo plano en su dispersión lateral de los rayos reflejados. Por esta razón, el ancho máximo de los pétalos debe ser menor que el diámetro del objeto sobre el que se desea concentrar la energía solar. Éste debe ser el criterio para planificar la cantidad de pétalos, la cual, obviamente, dependerá del radio máximo del paraboloide. Por ejemplo, si el objeto sobre el que deben converger los rayos tiene un diámetro de $0,2 \mathrm{~m}$, el ancho máximo de los pétalos debe ser menor, o a lo sumo igual a esta longitud. Si se planea hacer un paraboloide de $1 \mathrm{~m}$ de diámetro (lo mínimo recomendable, por ejemplo, para una cocina solar), se tendrá una circunferencia máxima de aproximadamente $3,14 \mathrm{~m}$ y la cantidad de pétalos necesaria será $n=3,14 / 0,2=15,7 \approx 16$ pétalos.

\subsubsection{Aprovechamiento del área de la lámina reflectante}

Los materiales reflectantes flexibles suelen comercializarse, al por mayor, en rollos, y se venden al consumidor final en láminas rectangulares o cuadradas. El método más 
usado para construir un paraboloide en pétalos consiste en imprimir un diagrama completo de pétalos sobre un pliego de papel, y luego pegarlo a una lámina reflectante, rectangular o cuadrada, para recortarla siguiendo este diagrama. Al proceder de esta manera, no se aprovecha toda el área de la lámina. La Fig. 4a muestra que al superponer un diagrama de pétalos completo (resaltado en azul claro) sobre una lámina cuadrada, se aprovecha sólo un sector central de la misma, pero se desaprovecha el área de los vértices, que es aproximadamente un $27 \%$ del área de una lámina cuadrada. (En adelante, llamaremos "convencional" a este diseño.)

Si, por el contrario, sobre una lámina cuadrada o rectangular, se superpone una fracción del diagrama en pétalos, de igual forma y tamaño que la lámina, como la resaltada en azul claro en la Fig. 4b, se aprovecha prácticamente toda el área reflectante. (En adelante, llamaremos "optimizado" a este diseño.)

Sin embargo, en el diseño optimizado, a diferencia del convencional, al unir los bordes de los pétalos, éstos no quedan en un mismo plano con el eje del paraboloide, y sufrirán tensiones oblicuas que no serán iguales para todas los pétalos ni serán simétricas en cada borde de un mismo pétalo. Por esta razón, este diseño sólo debe aplicarse en láminas muy flexibles, de tal modo que los pétalos al curvarse generen una sección aproximadamente circular; o bien usar diagramas de muchos pétalos, de tal modo que el ancho máximo de éstos sea bastante pequeño, y la dispersión lateral oblicua de los rayos reflejados quede acotada dentro de un entorno próximo al foco. Con estas precauciones, para un dado tamaño de la lámina reflectante, el diseño optimizado puede ofrecer un rendimiento bastante mayor que el convencional. La
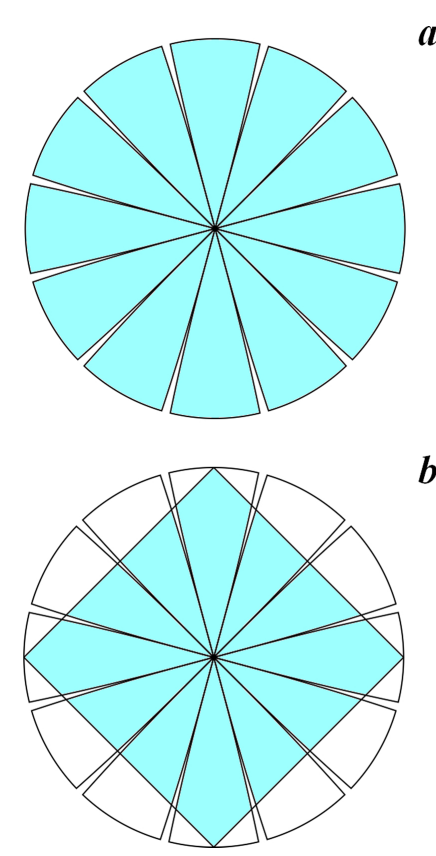

Figura 4: Diagrama de pétalos de los diseños convencional (a) y optimizado (b). superioridad en el rendimiento dependerá de la distancia focal del reflector, como se discute en la siguiente sección.

\subsubsection{Planificación de la distancia focal}

2.4.3.1. Criterios para elegir una distancia focal Hay dos aspectos muy importantes en la planificación de la distancia focal: la seguridad y la eficiencia. Los paraboloides con foco interno, es decir, aquellos en que el foco está en el interior del espacio cóncavo del paraboloide, son más seguros a los fines de evitar deslumbramientos o quemaduras accidentales, pero las distancias focales pequeñas son poco eficientes, al igual que las demasiado grandes. Si la distancia focal es demasiado grande, el objeto a ser irradiado deberá colocarse relativamente lejos de la superficie del reflector, quedando más expuesto a pérdidas de energía térmica a través de brisas o corrientes convectivas.

$\mathrm{Si}$, por el contrario, la distancia focal es pequeña, el aprovechamiento de la superficie reflectante es muy limitado, como se explica en el siguiente ejemplo:

Supóngase que se dispone de una lámina cuadrada de $1 \mathrm{~m}$ de lado y se decide usar el diseño convencional (Fig. 4a) para construir el paraboloide. Se elige una distancia focal $f=0,25 \mathrm{~m}$, y una longitud máxima $L_{\max }=0,5 \mathrm{~m}$ para los pétalos, a fin de aprovechar la máxima superficie que permite este diseño. Al reemplazar estos valores en la Ec. 3' y resolverla numéricamente ${ }^{4}$ se obtiene un valor máximo para el radio: $\rho_{\max } \cong 0,4463 \mathrm{~m}$. Suponiendo una sección circular, el área efectiva será $A_{e f}=\pi \rho_{\max }^{2} \cong$ $0,6258 \mathrm{~m}^{2}$. El área efectiva es la proyección del área del paraboloide en un plano normal a su eje (es decir, el área de la sombra del paraboloide, en un plano normal a su eje, cuando éste está dirigido hacia el sol). Este parámetro es proporcional a la cantidad de radiación que incide en el paraboloide.

El mismo cálculo, para una distancia focal de $1 \mathrm{~m}$, da un radio máximo de aproximadamente 0,4949 m y un área efectiva de aproximadamente $0,7694 \mathrm{~m}^{2}$. Luego, al aumentar la distancia focal de 0,25 m a $1 \mathrm{~m}$, el área efectiva aumenta casi un $23 \%$, y por tanto el paraboloide puede captar una cantidad de energía un $23 \%$ mayor que la del caso anterior.

Desde luego, el hecho de que un diseño de reflector capte más energía que otro no necesariamente implica que sea más eficaz, pues no toda la energía captada se aprovecha. El aprovechamiento de energía será tanto mayor cuanto mayor sea la absorción del objeto irradiado y cuanto menor sea su pérdida térmica. La dispersión de los rayos reflejados, debida fundamentalmente a los defectos de construcción, también afecta el rendimiento. En este sentido es importante destacar que la distancia focal asume un doble compromiso, pues si bien una distancia focal grande implica una mayor distancia entre el reflector y el objeto irradiado, y esto contribuye a

\footnotetext{
${ }^{4}$ La Ec. 3 es una ecuación trascendente que no se puede resolver en forma analítica.
} 
aumentar su pérdida térmica, el aumento de la distancia focal, además de aumentar el área efectiva, contribuye a disminuir los efectos dispersivos, como se analizará en $\$ 3.4$ (esta sección está referida al modelo de reflector catenoide, pero los resultados sobre la distancia focal son totalmente aplicables al paraboloide).

\subsubsection{Contribución de los vértices de la lámi-} na al área efectiva y efecto de la distancia focal Consideremos ahora el diseño optimizado (Fig. 4b). Por brevedad, analizaremos sólo el caso de una lámina cuadrada, pero la extensión al caso de una lámina rectangular es muy simple e intuitiva. Dada la simetría de la Fig. 4b, a los fines de calcular el área efectiva, basta considerar un solo cuadrante. La Fig. 5 es una ampliación del cuadrante superior derecho de la Fig. 4b. La línea roja representa un lado de la lámina cuadrada, superpuesta en forma oblicua al diagrama de pétalos, y se ha marcado un segmento (línea azul), desde el origen hasta esta línea roja. Este segmento azul, de longitud $L_{h}$, forma un ángulo $\varphi$ con la horizontal, y su extremo superior tiene coordenadas $x_{h}$ y $y_{h}$.

Por el teorema de Pitágoras, se cumple

$$
L_{h}^{2}=x_{h}^{2}+y_{h}^{2}
$$

donde $x_{h}$, a su vez, cumple con la ecuación

$$
x_{h}=L_{h} \cos (\phi)
$$

Por otra parte, el segmento rojo, que representa un lado de la lámina, es parte de una recta de ecuación

$$
y=-x+C
$$

Donde la ordenada al origen $C$ es la mitad de la diagonal de la lámina (por ejemplo, para una lámina de $1 \mathrm{~m}$ de lado, como en los ejemplos anteriores, se tiene $C=\sqrt{2} / 2$ $\mathrm{m})$.

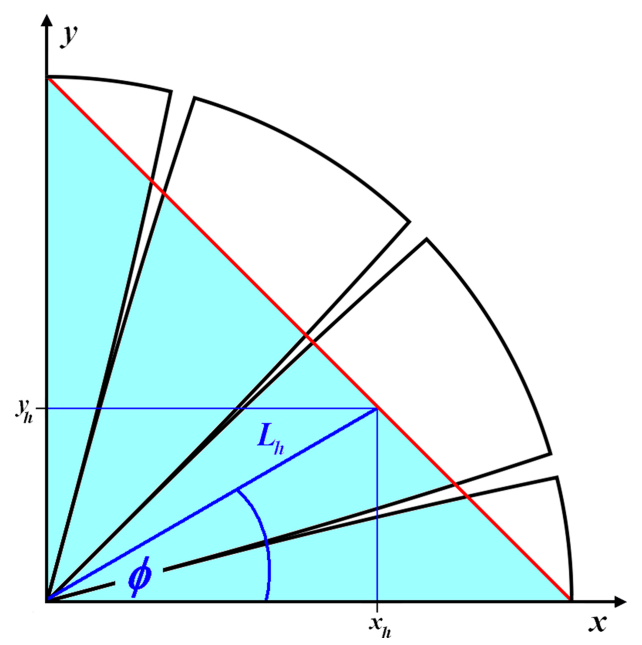

Figura 5: Ampliación del cuadrante superior derecho de la Fig. $4 \mathrm{~b}$, donde se indica un lado de la lámina reflectante (segmento rojo) y las coordenadas de un punto genérico sobre dicho lado.
Las Ecs. (8), (9) y (10) constituyen un sistema que permite obtener $L_{h}$ en función de $\varphi$. Este cálculo teórico es aplicable a cualquier número de pétalos.

Una vez encontrada una dada longitud $L_{h}$, la Ec. (3') permite encontrar el correspondiente radio $\rho$ del paraboloide, cuyas coordenadas cartesianas, en un plano normal al eje del paraboloide, serán

$$
\begin{aligned}
& x_{r}=\rho \cos (\phi) \\
& y_{r}=\rho \operatorname{sen}(\phi)
\end{aligned}
$$

Las Ecs. 111 y (12) son las coordenadas del borde de la sección eficaz en un plano normal al eje del paraboloide (o lo que es lo mismo, las coordenadas del borde de la sombra del paraboloide en un plano normal a su eje, cuando éste apunta hacia el sol). Una vez obtenidas las coordenadas del borde de la sección eficaz, se puede obtener el área de la misma por integración numérica. En [12] ofrecemos un programa de nuestra autoría, Programa II, desarrollado en Scilab 5.5.1, que calcula secciones eficaces de paraboloides y la contribución relativa de los vértices de la lámina reflectante, para valores arbitrarios del tamaño de la misma y de la distancia focal. Dicho programa también grafica la forma de la sección eficaz.

La Fig. 6 representa dos paraboloides de diseño optimizado (paneles $a$ y $c$ ), de sección circular, y sus correspondientes secciones eficaces (paneles $b$ y $d$ ). En ambos casos la lámina reflectante es cuadrada y su lado tiene $1 \mathrm{~m}$ de longitud. Los paneles $a$ y $b$ corresponden a una distancia focal de $0.25 \mathrm{~m}$; y los paneles $c$ y $d$, a una distancia focal de $1 \mathrm{~m}$. El círculo central marcado en todos los paneles es igual a la sección máxima del diseño convencional (Fig. 4a), cuyos parámetros han sido calculados en la sección §2.4.3.1, para las dos distancias focales de nuestro ejemplo.

De esto se infiere que tanto el diseño convencional como el optimizado (Figs. 4a y 4b) conducen al mismo tipo de paraboloide, excepto porque en el diseño optimizado, la sección eficaz es mayor debido a que contiene el área adicional de los vértices, anotada en los paneles $b$ y $d$ de la Fig. 6. Para distancias focales pequeñas, la contribución de los vértices es menor, tanto en términos absolutos como relativos. En nuestro ejemplo comparativo, con una lámina de $1 \mathrm{~m}$ de lado y una distancia focal de 0,25 $\mathrm{m}$, la sección eficaz de los vértices es $\sim 0,134 \mathrm{~m}^{2}$, en tanto que para el mismo tamaño de lámina y una distancia focal de $1 \mathrm{~m}$, es $\sim 0,204 \mathrm{~m}^{2}$. En términos relativos, el aumento de la sección eficaz debido a la contribución de los vértices es $\sim 21,41 \%$ en el primer caso, $\mathrm{y} \sim 26,52 \%$ en el segundo.

Como puede apreciarse en los paneles $b$ y $d$ de la Fig. 6, cuando la distancia focal es menor, la proyección de los vértices tiene lados más redondeados. Esto es coherente con el hecho de que su contribución relativa a la sección eficaz sea menor, como se muestra en la Fig.7, pues cuanto más redondeados sean los lados, más se aproximarán al círculo central y menor será el área que 


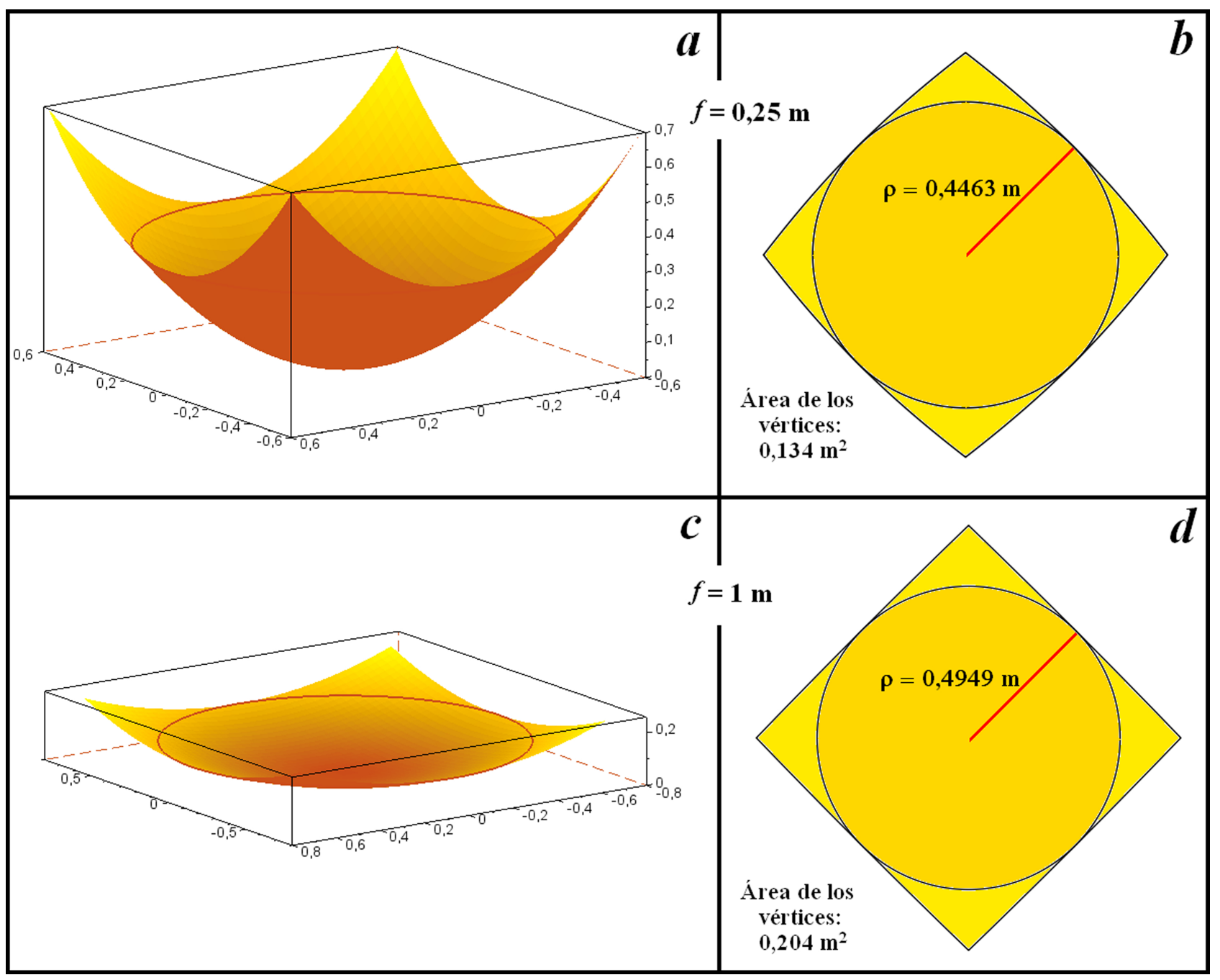

Figura 6: Dos paraboloides de diseño optimizado (paneles a y c) y sus correspondientes secciones eficaces (paneles b y d). En ambos casos la lámina tiene un metro de lado. Se anotan en cada caso, la distancia focal, la contribución de los vértices al área efectiva, y el radio del círculo central que se corresponde con la sección máxima del diseño convencional.

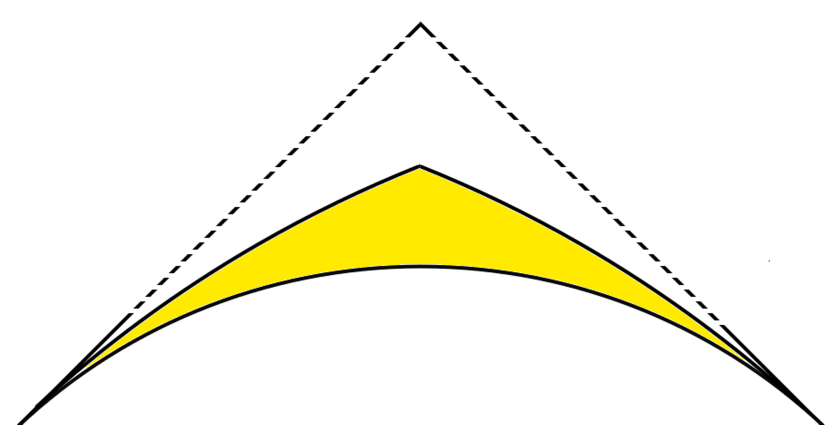

Figura 7: Área eficaz de un vértice correspondiente a una distancia focal pequeña (superficie amarilla de lados superiores redondeados) y limite para una distancia focal infinita (líneas rectas de trazos).

encierren. Además, debe tenerse presente que el radio del círculo central disminuye con la distancia focal.

Si se calcula cuánto contribuyen los vértices al área de la sección eficaz, para valores arbitrariamente pequeños o grandes de la distancia focal (v. Programa II en [12]), se comprueba que esta contribución relativa varía en un intervalo acotado, desde $\sim 12,22 \%$ para distancias focales extremadamente pequeñas hasta $\sim 27,32 \%$, para distancias focales extremadamente grandes. Esto es coherente con el hecho de que cuando la distancia focal tiende a infinito, el paraboloide tiende a un plano (v. Fig. 6, paneles $c$ y $d$ ). En un plano, el cociente entre el área total de un cuadrado y la del círculo central inscrito en él es $L^{2} / \pi(L / 2)^{2}=4 / \pi \cong 1,273239$ Luego, el área de un cuadrado es $\sim 27,32 \%$ mayor que el área de un círculo inscrito en él, y éste es el límite al que tiende la contribución relativa de los vértices cuando la distancia focal tiende a infinito 


\section{Segunda Parte: El Reflector Catenoide}

\subsection{Fundamentos geométricos}

En esta sección presentamos un nuevo modelo de reflector de bajo costo que, en nuestro conocimiento, no ha sido tratado en la literatura especializada: el catenoide ${ }^{5}$

El catenoide es la superficie de revolución cuya generatriz es una catenaria. Ésta es la curva que describe una cadena, cuerda o cable inextensible, de densidad lineal uniforme y rigidez flexional depreciable, bajo la acción de su propio peso, cuando se suspende de sus extremos, en un campo gravitatorio uniforme. Estas condiciones no son tan restrictivas como pueden parecerlo a primera vista, y muchos materiales las cumplen de manera satisfactoria. En particular, un sistema de gran tamaño podría construirse con un material relativamente rígido y aun así adoptar una forma que, para todos los fines prácticos, podría considerarse una catenaria. 6

El mismo tipo de análisis desarrollado para el paraboloide, en las secciones $\$ 2.2$ y $\S 2.3$, es aplicable al catenoide, es decir, se puede diseñar y construir en pétalos. Si bien en superficies de revolución ideales, el catenoide no enfoca los rayos reflejados con tanta precisión como el paraboloide, en los diseños de pétalos, ambos tienen una precisión comparable. Esto se debe a que, en el paraboloide, el ancho de las pétalos suele provocar una mayor dispersión lateral de enfoque porque, en general, se construye con materiales más rígidos, para mantener su forma (v. §2.4.1).

El catenoide debe construirse con materiales mucho más flexibles y delgados (en $\$ 5.2$ se sugieren algunos materiales apropiados) porque la forma buscada es la que adopta el sistema cuando se deja librado a su propio peso, desde un conjunto de puntos de suspensión. El mero hecho de trabajar con este tipo de materiales hace que la tarea de corte y armado sea más sencilla, permitiendo una mayor prolijidad y, por tanto, una mayor eficacia del reflector. O bien, si no se requiere una gran precisión, el corte de los pétalos se puede hacer en forma aproximada y no es necesario hacer el ensamblado, porque cada pétalo adoptará la forma apropiada por la simple acción de su propio peso (esta posibilidad se analiza en §3.3.1). Otras ventajas del catenoide, respecto del paraboloide, son: $a$ ) Las ecuaciones necesarias para el diseño son más simples y todas se pueden resolver analíticamente. b) No se requiere diagramas de muchos pétalos, pues el sistema tiende

\footnotetext{
5 En algunos contextos, se llama "catenoide" a la superficie de revolución generada por una catenaria cuando gira respecto del eje de las abscisas, y "casa" (house) a la superficie generada por una catenaria cuando gira respecto del eje de simetría (eje de las ordenadas). Sin embargo esta nomenclatura no está muy extendida, y nos parece más intuitivo y coherente con las convenciones geométricas y lingüísticas usar el término "catenoide" para cualquiera de los dos casos.

${ }^{6}$ Los cables de acero de los puentes colgantes, por ejemplo, adoptan una forma intermedia entre una catenaria y una parábola, entre las cuales hay muy poca diferencia, como se discute más adelante.
}

a adoptar por sí mismo una sección aproximadamente circular. c) El reflector se puede enrollar o plegar para guardarlo en poco espacio. Todas estas características hacen que este modelo sea idóneo para construir sistemas de gran tamaño, lo cual implica mayor potencia en la concentración de energía.

A nuestro parecer, la principal desventaja de este modelo es que no se puede orientar hacia el sol (tal como lo planteamos en este trabajo, el eje del catenoide se mantiene siempre vertical). En zonas y épocas del año en que la declinación solar es leve, ésta no es una desventaja muy significativa, y puede verse compensada con creces en sistemas de gran tamaño. Con un diseño mucho más complicado, se podría orientar el eje en otra dirección, pero esto no aportaría mayor beneficio, porque seguiría siendo prácticamente imposible hacer un seguimiento del recorrido del sol. Esta desventaja ocasionada por la incidencia oblicua de los rayos solares se discute en la sección §3.4.

\subsection{La catenaria: curva generatriz}

La ecuación de una catenaria con vértice en el origen puede escribirse

$$
z=2 f\left[\cosh \left(\frac{x}{2 f}\right)-1\right]
$$

Si bien la catenaria no tiene foco ni distancia focal, hemos incluido en la Ec. (13) el parámetro $f$ a los fines de comparar esta curva con una parábola cuyo vértice está en el origen (Ec. (1)).

El desarrollo en serie de Maclaurin de $\cosh (x)$ es [13]

$$
\cosh (x)=1+\frac{x^{2}}{2 !}+\frac{x^{4}}{4 !}+\frac{x^{6}}{6 !} \ldots
$$

De las Ecs. 13) y 14 se obtiene

$$
z=\frac{x^{2}}{4 f}+O\left(x^{4}\right)
$$

Lo cual implica que, en el entorno del origen, la catenaria (Ec. (13) tiene un comportamiento muy similar a la parábola (Ec. (1)).

La Fig. 8 muestra las gráficas de las Ecs. (1) y (13) (curvas roja y verde, respectivamente) para $f=1$, en un intervalo de abscisas desde $x_{\text {min }} \cong-0,69 \mathrm{~m}$ hasta $x_{\text {max }} \cong 0,69 \mathrm{~m}$. Sobre ellas incide verticalmente un haz de rayos paralelos (flechas amarillas). Por claridad, en el semiespacio negativo de las abscisas sólo se ha graficado el haz reflejado en la catenaria (segmentos verdes), y en el semiespacio positivo, sólo el reflejado en la parábola (segmentos rojos).

Se observa que, dentro del grado de resolución de la figura, las curvas de la párabola y de la catenaria están superpuestas. Todos los rayos reflejados en la parábola convergen al foco, como cabe esperar; mientras que los reflejados en la catenaria muestran un desenfoque en altura, el cual es progresivamente mayor cuanto más alejado 


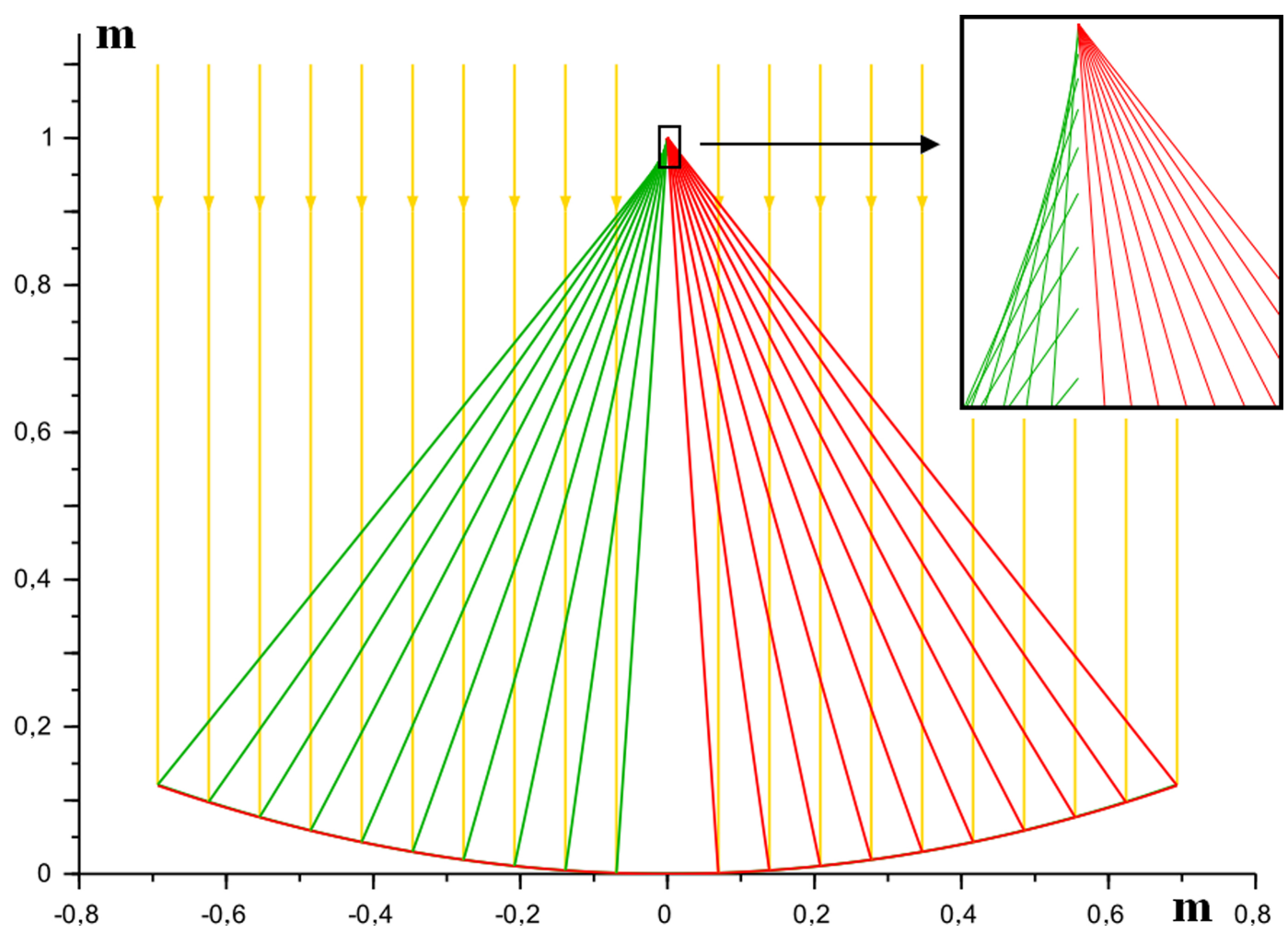

Figura 8: Diagrama de reflexión de un haz de rayos paralelos (flechas amarillas) que inciden sobre una parábola y una catenaria (curvas indistinguibles en el grado de resolución de la figura). Los segmentos rojos representan los rayos reflejados en la parábola, y los verdes, los reflejados en la catenaria.

del origen está el punto de incidencia. Sin embargo, para este particular valor de $f$ y en este particular intervalo de abscisas, se tiene que la dispersión de los rayos reflejados está acotada en un intervalo de altura de $\sim 0,012 \mathrm{~m}$. Para valores menores de $f$, o intervalos mayores de abscisas, la dispersión sería mayor.

Al igual que en el caso de la parábola, también en la catenaria la abscisa máxima se corresponde con el radio máximo del catenoide, y es posible dimensionar este radio y la distancia focal de tal modo que la dispersión longitudinal de los rayos reflejados quede acotada en un intervalo aceptable, y todos converjan en el objeto que se desee irradiar. Para el conjunto de valores de la Fig. 8, por ejemplo, bastaría que el objeto tenga una longitud mayor o igual a $\sim 0,012 \mathrm{~m}$ (cualquiera sea su diámetro), o un diámetro mayor o igual a $\sim 0,007 \mathrm{~m}$ (cualquiera sea su longitud). Obviamente, estas condiciones son muy poco restrictivas.

Calculando el paréntesis del último miembro de la Ec. (2) a partir de la Ec. (13) e integrando, se tiene que la longitud del arco de catenaria comprendido entre el origen (vértice de la catenaria) y un punto cualquiera $P(x, z)$ perteneciente a ella está dada por

$$
L=2 f \cdot \operatorname{senh}\left(\frac{x}{2 f}\right)
$$

Y dado que una abscisa $x$ de la catenaria corresponde a un radio $\rho$ del catenoide, conviene reescribir esta ecuación en términos del radio:

$$
L=2 f \cdot \operatorname{senh}\left(\frac{\rho}{2 f}\right)
$$

La Ec 17 permite despejar $\rho$ en términos analíticos (a diferencia de la Ec. (3), su homóloga en el caso del paraboloide). Esto facilita los cálculos de diseño.

Para los pétalos del catenoide también se aplican las Ecs. (5), (6) y (7), que junto con la Ec. (17) permitirán obtener las coordenadas del borde lateral de un pétalo, para graficar el diagrama a escala con la precisión deseada.

En [12] se ofrece un programa de nuestra autoría, Programa III, desarrollado en Scilab 5.5.1, para graficar con precisión diagramas de pétalos para un catenoide. Este programa cuenta con las mismas prestaciones mencionadas en el caso del paraboloide.

\subsection{Diseños de reflectores catenoides}

\subsubsection{Diseño sencillo}

En este diseño no es necesario recortar los pétalos con mucha prolijidad, ni tampoco ensamblarlos; sólo se debe calcular con precisión los puntos de suspensión. Si éstos están bien ubicados, aunque los bordes de los pétalos no estén unidos entre sí, cada pétalo adoptará, por su propio peso, la forma de un casquete de catenoide. Por otra parte, si los pétalos se recortan un poco más anchos de lo que 
son, es decir, dejando pequeños rebordes laterales, los rebordes de pétalos contiguos se solaparán entre sí, pero ello no afectará apreciablemente la estructura general.

A modo de ejemplo, consideremos el diagrama de la Fig. 9, que consta de ocho pétalos representados alternadamente en colores marrón y amarillo. Con este diagrama puede construirse un catenoide como el representado en la Fig. 10 (vista superior), donde los puntos de suspensión se han marcado con pequeños círculos azules. Éstos son los únicos puntos de ensamblaje entre pétalos contiguos y deben ser tantos como pétalos tenga el diseño. Si esta cantidad es par, los puntos de suspensión estarán enfrentados, de a pares, en puntos diametralmente opuestos del catenoide.

Supongamos que elegimos una distancia focal de $1 \mathrm{~m}$ y la longitud de cada pétalo de la Fig. 9 es $L=0,5 \mathrm{~m}$. De la Ec. (17) se obtiene que el radio del catenoide que corresponde a esta longitud es $\rho_{L} \cong 0,495$; y de la Ec. (13), reemplazando $x$ por $\rho_{L}$, se obtiene que la altura que corresponde a este radio es $z_{\rho} \cong 0,062 \mathrm{~m}$. Luego, los puntos de suspensión deben estar enfrentados, de a pares, a distancias de $2 \rho_{L}$, y deben estar todos a una misma altura $z_{s}$, tal que $z_{s} \geq z_{\rho}$. Si $z_{s}=z_{\rho}$, el centro o vértice del catenoide (punto verde central en la Fig. 10) rozará el suelo, y si $z_{s}>z_{\rho}$, el vértice quedará a una altura del suelo igual a la diferencia entre estos dos valores.

Los vértices superiores de cada pétalo sí deben recortarse con prolijidad, a fin de que dos vértices contiguos puedan compartir el mismo punto de suspensión sin provocar deformaciones. Por otra parte, los cortes laterales de los pétalos no deben hacerse hasta el centro mismo del diagrama, sino hasta el punto en que dos lados adyacentes se confunden en una sola línea. Esto dará continuidad a todo arco del catenoide que se extienda en un plano

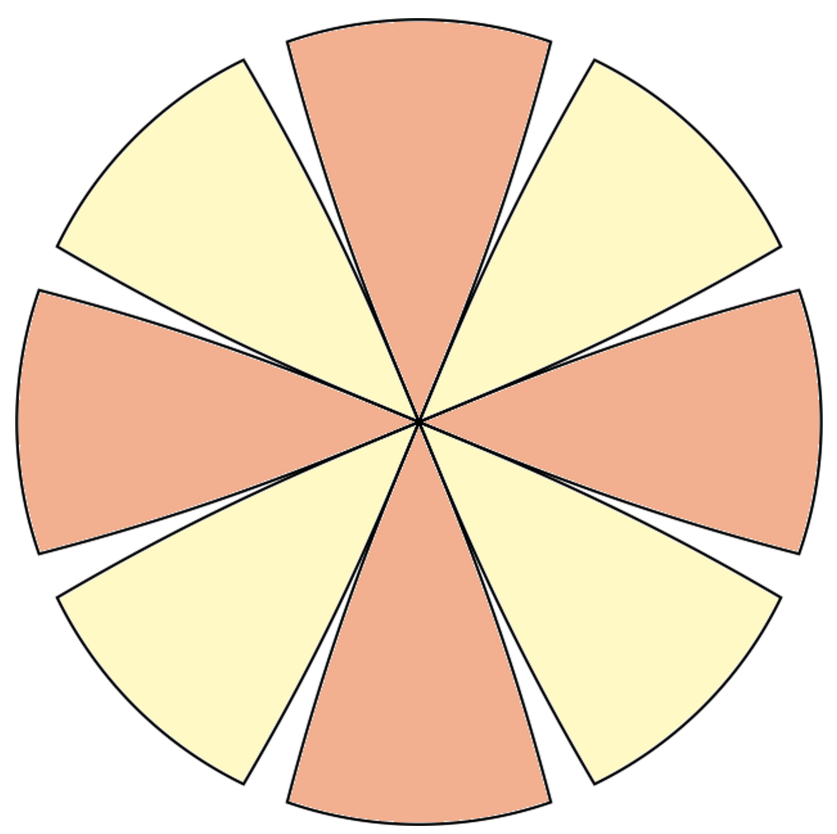

Figura 9: Diagrama de pétalos de un catenoide de diseño sencillo.

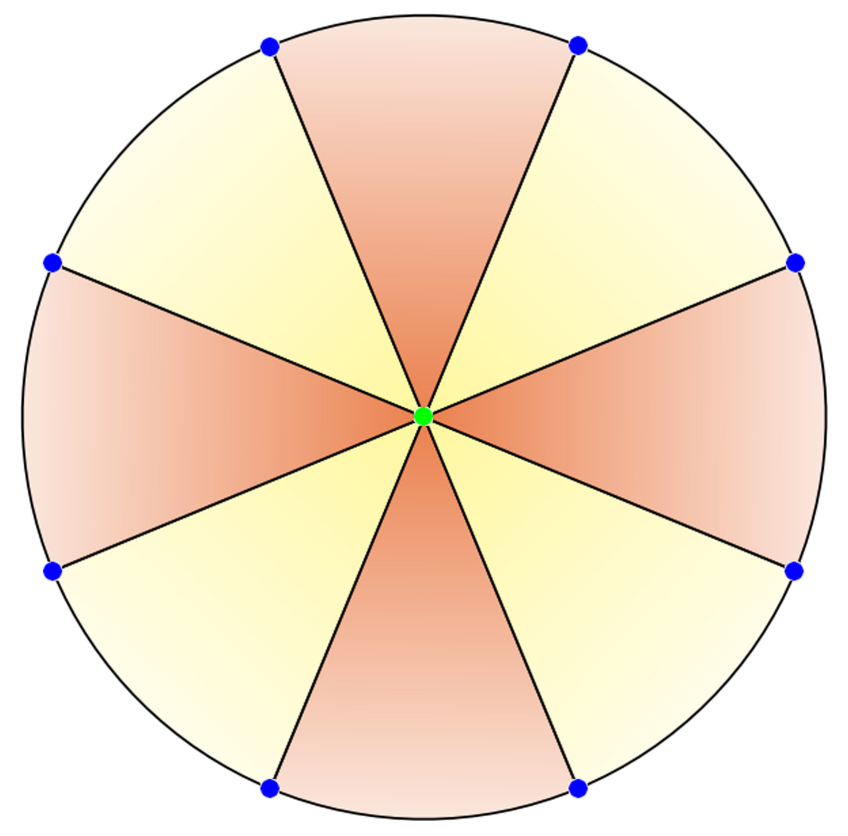

Figura 10: Vista superior de un catenoide construido con el diagrama de la Fig. 9. Los círculos azules representan los puntos de suspensión, y el círculo verde representa el vértice del catenoide (punto de altura mínima).

vertical, pasando por el vértice del mismo. Cualquiera de estos arcos será una catenaria cuyo punto medio será el vértice del catenoide, y éste recibirá tensiones iguales en todas las direcciones.

\subsubsection{Diseño optimizado}

En este diseño, la cantidad de pétalos debe ser un múltiplo de cuatro. Tomaremos como ejemplo el diagrama de ocho pétalos representado en la Fig. 11, al que se le ha superpuesto una lámina cuadrada, resaltada en azul claro, aplicando el mismo criterio planteado para un paraboloide en la Fig. 4b (v. §2.4.2).

Luego de efectuar los cortes y el ensamblado, la lámina debe suspenderse de sus cuatro vértices, desde puntos de sujeción enfrentados diagonalmente. De esta manera, las tensiones a lo largo de las diagonales se compensarán por simetría, y cada diagonal describirá una catenaria. En el espacio entre las diagonales las tensiones laterales sobre cada pétalo serán simétricas, y la lámina en su conjunto adoptará la forma aproximada de un catenoide de sección circular.

La ubicación apropiada de los puntos de suspensión se obtiene calculando la altura de los mismos y la distancia entre cada par de puntos enfrentados a lo largo de cada diagonal. Supongamos que tanto la distancia focal como el lado de la lámina cuadrada miden $1 \mathrm{~m}$. Luego, la longitud desde el centro de la lámina hasta cualquiera de sus vértices es la mitad de la diagonal, i. e., $\sqrt{2} / 2 \mathrm{~m}$. Este valor es también la longitud $L_{v}$ del tramo de catenaria comprendido entre el origen y el radio $\rho_{v}$ que deberá tener el catenoide en cada uno de los cuatro vértices de 


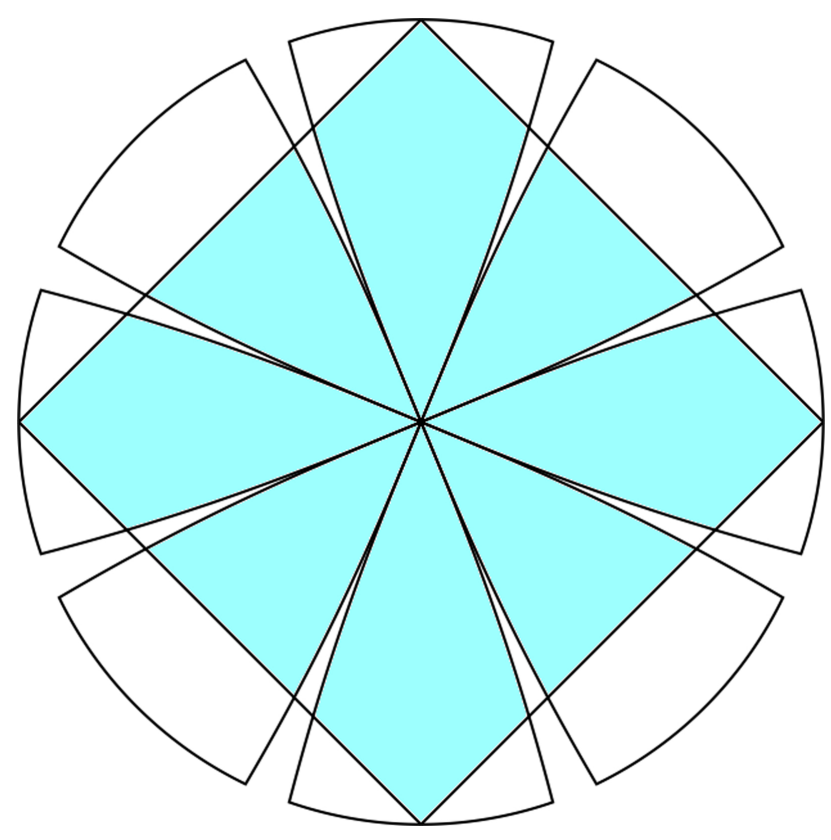

Figura 11: Diagrama de pétalos al que se le superpuesto una lámina cuadrada (resaltada en azul claro) para construir un catenoide de diseño optimizado

la lámina (Ec. (17)). Luego, tomando $L_{v}=\sqrt{2} / 2$ en la Ec. 17) se obtiene el valor $\rho_{v} \cong 0,693 \mathrm{~m}$, y reemplazando este valor en la Ec. (13) se obtiene $z_{v} \cong 0,121 \mathrm{~m}$.

Así, cada par de puntos de suspensión enfrentados a lo largo de una diagonal deben estar a una distancia de $2 \rho_{v}$, y todos los puntos deben estar a una altura $z_{v}$, o mayor, según se prefiera que el vértice del catenoide roce el suelo o quede elevado a una cierta altura

Si se calcula cuánto contribuyen los vértices de una lámina cuadrada al área efectiva del catenoide, para distancias focales comparables o mayores que la diagonal de la lámina (requisito de buena convergencia), los resultados son prácticamente los mismos que los obtenidos en el caso de un paraboloide. Este cálculo se puede hacer con un programa de nuestra autoría ofrecido en [12] Programa IV, el cual también grafica el área efectiva de este diseño.

\subsection{Incidencia oblicua de los rayos solares}

Como se adelantó en la sección §3.1, el hecho de que el catenoide no pueda orientarse hacia el sol disminuye su rendimiento como reflector Esto se debe a que, bajo incidencia oblicua el área efectiva se reduce y la dispersión de los rayos reflejados aumenta.

El área efectiva se reduce en un factor $\cos (\alpha)$, donde $\alpha$ es el ángulo entre la dirección de los rayos solares y el eje del reflector (vertical). La mayor dispersión, por su parte, está asociada con un aumento y un desplazamiento del área de convergencia, y depende de $\alpha$ y de la distancia focal del reflector

La Fig. 12 muestra estos dos tipos de dependencia. En los paneles superiores $(a, b$ y $c$ ) se representa una catenaria con $f=1,5$ (Ec. $(13))$, sobre la que inciden haces de rayos oblicuos: en $a \alpha=10^{\circ}$; en $b \alpha=20^{\circ}$; y en $c \alpha=30^{\circ}$. En el vértice superior izquierdo de cada panel se anota el valor del $\cos (\alpha)$ correspondiente a cada uno de estos ángulos, lo cual representa el factor de reducción del área efectiva. Así, por ejemplo, para $\alpha=10^{\circ}$, el área efectiva equivale al 98,5\% del área efectiva total que se tendría bajo incidencia vertical. De esto se deduce que la reducción del área efectiva es relativamente moderada si $\alpha$ no es demasiado grande y en un sistema de gran tamaño esto no representa una desventaja crítica.

También se observa que, a medida que $\alpha$ crece, la dispersión aumenta y el centro de la zona de convergencia se desplaza cada vez más a la derecha (en dirección opuesta al sol) y hacia abajo. Esto sugiere que desplazando el objeto que se desea irradiar, se puede soslayar en buena medida esta desventaja. Debe advertirse que no es necesario que el tamaño ni la forma del objeto sean comparables a los de la zona de convergencia. Basta que al menos una de sus dimensiones (longitud o diámetro) permita que todos los rayos reflejados en un dado rango incidan sobre él. Así, por ejemplo, en el caso $c$, que es el más desfavorable de los tres, un objeto de $\sim 0,5 \mathrm{~m}$ de longitud vertical que se colocara con su base a una altura de $1 \mathrm{~m}$ y a $0,8 \mathrm{~m}$ a la derecha del eje del catenoide, interceptaría la trayectoria de todos los rayos reflejados, sin importar cuál fuera su diámetro. Lo mismo sucedería con un objeto de $\sim 0,5 \mathrm{~m}$ de diámetro que se colocara $\mathrm{a} \sim 0,7 \mathrm{~m}$ de altura, con su base centrada a $\sim 0,55 \mathrm{~m}$ a la derecha del eje, sin importar cuál fuera su longitud vertical. Así, para un objeto cualquiera, con una dada longitud y un dado diámetro, en general podrá encontrarse una posición donde éste sea bien irradiado. Una pequeña ventaja colateral de este método es la de disminuir o eliminar la sombra del objeto sobre el reflector, la cual, en algunos casos, puede disminuir el área efectiva tanto o más que la incidencia oblicua.

En los paneles $d$, e y $f$, se representan catenarias de distintas distancias focales: $1 \mathrm{~m}, 1,5 \mathrm{~m}$ y $2 \mathrm{~m}$, respectivamente (en todos estos casos, $\alpha=10^{\circ}$ ). Como puede apreciarse, la dispersión de los rayos reflejados disminuye notablemente cuando la distancia focal aumenta. Esto enfatiza la conveniencia de construir sistemas de distancias focales relativamente grandes, en concordancia con otras ventajas ya discutidas en la sección §2.4. El desplazamiento del centro de la zona de convergencia en dirección opuesta al sol varía en forma comparable al de la dependencia angular (paneles superiores $a, b, c$ ). El desplazamiento hacia abajo del centro de esta zona es esencialmente el mismo para las tres distancias focales.

Si bien este análisis, por brevedad, se ha realizado en dos dimensiones, su extensión a tres dimensiones es relativamente sencilla y bastante intuitiva. ${ }^{7}$ En [12] ofre-

\footnotetext{
7 Debe notarse que un plano vertical que contenga a la dirección de los rayos solares y al eje de un catenoide de revolución divide a éste en dos mitades. Entre ellas habrá una completa simetría de los rayos incidentes y reflejados, de tal modo que estos últimos
} 


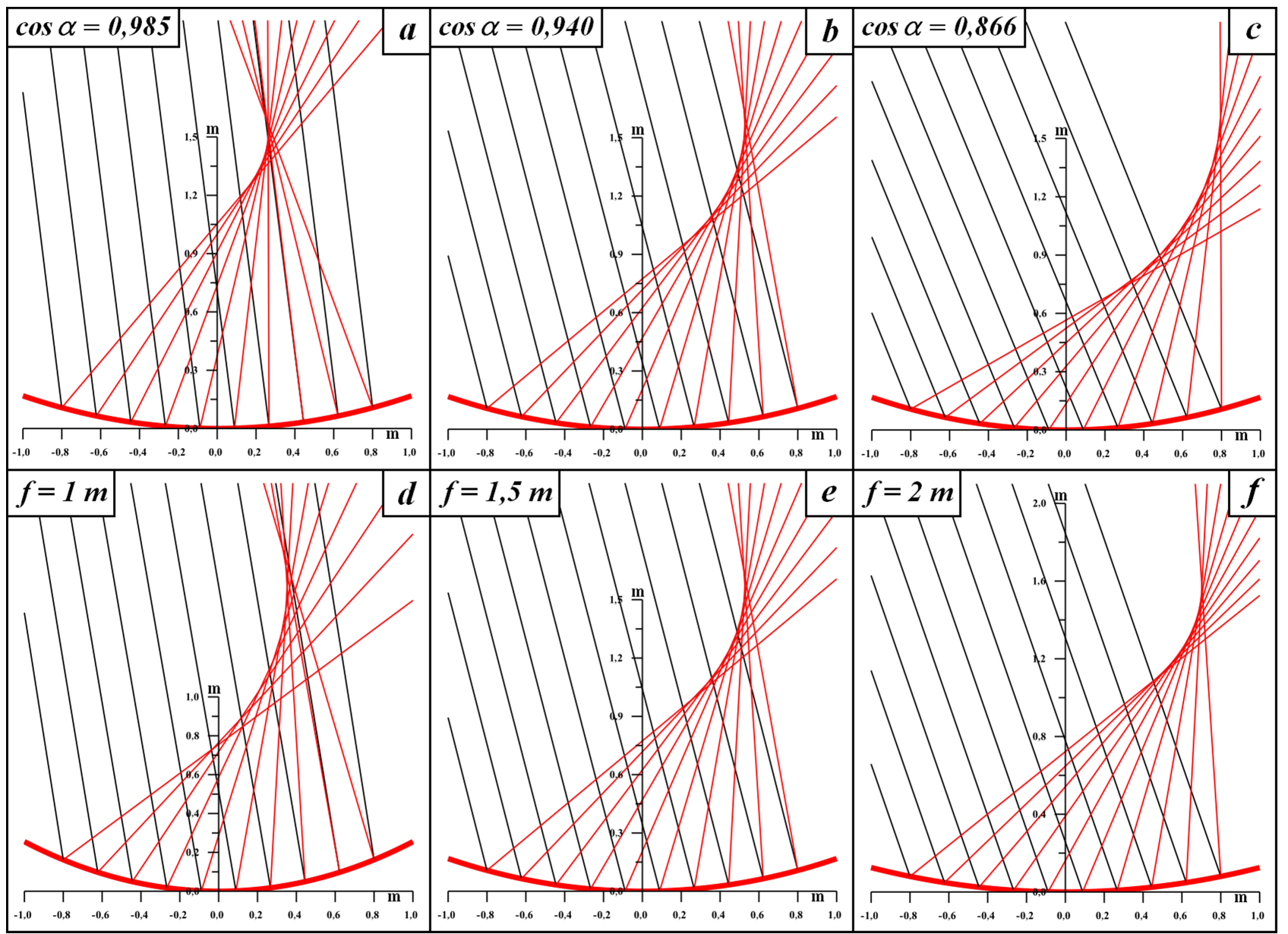

Figura 12: Incidencia oblicua en un catenoide. Paneles superiores ( $a, b, c)$ : Efecto del ángulo $\alpha$ entre los rayos solares y el eje del reflector. Paneles inferiores ( $\mathrm{d}, \mathrm{e}, \mathrm{f})$ : Efecto de la distancia focal $f$.

cemos un programa de nuestra autoría, Programa V, que permite calcular gráficas como las de la Fig. 12, donde el usuario puede elegir el valor del ángulo $\alpha$ y de la distancia focal.

\section{Criterios para Elegir las Dimensiones del Reflector}

Cualquiera sea el modelo de reflector que se elija construir, debe decidirse un tamaño del mismo adecuado a sus fines Si éstos son didácticos, el tamaño no es un factor restrictivo, pues basta que los estudiantes puedan apreciar cualitativamente el comportamiento del sistema y eventualmente hacer mediciones relativamente sencillas. En caso de que la construcción se plantee como proyecto experimental, puede convenir un tamaño moderado que permita a los estudiantes trabajar con facilidad y evitar mayores costos.

Si por el contrario, el reflector será destinado a una aplicación práctica tal como cocción de alimentos, calefacción o concentración de energía para usos diversos, el

se interceptan en el plano vertical mencionado, en la zona de convergencia descrita en nuestro análisis bidimensional. tamaño del sistema es un factor crítico, y su planificación, en general, no es un problema sencillo.

En principio, la potencia de un reflector es proporcional a su área efectiva y a la reflectancia del material usado. Sin embargo, la fracción aprovechable de esa potencia depende de otros factores muy diversos tales como el tamaño del objeto a irradiar, la distancia focal, los defectos de construcción y otros factores que inciden en la dispersión y pérdida de energía.

En general, tiene sentido construir un reflector de grandes dimensiones sólo cuando el objeto a irradiar también es grande. Si se coloca sobre un reflector grande un objeto pequeño, es muy probable que gran parte de los rayos reflejados no lleguen a incidir sobre él.

En reflectores grandes, el cociente entre la distancia focal y el diámetro del área efectiva suele ser menor que en reflectores pequeños, porque en general no conviene que el objeto esté demasiado lejos del reflector, ya que esto aumentaría su pérdida térmica. Esto implica, según lo discutido en $\S 2.4 .2$ y $\S 2.4 .3$, que cuanto mayor sea el reflector, el aprovechamiento del material disponible en términos del área efectiva y de la potencia, será relativamente menor, y se requerirá una gran cantidad de 
material para obtener sólo una pequeña mejora en el rendimiento. Por otra parte, las distancias focales pequeñas son más sensibles a los defectos de construcción y a las deformaciones de la superficie reflectante, como puede inferirse de la Fig. $12(d, e, f)^{8}$ pues éstos implican inclinaciones de la superficie que varían el ángulo de incidencia.

En el caso de un paraboloide, debe advertirse que un material que en pequeña escala es bastante rígido como para mantener su forma y soportar ciertos esfuerzos, en gran escala será más propenso a deformarse. Esto obliga a extremar los cuidados de mantenimiento, o bien, simplemente, a invertir en materiales de mayor espesor o rigidez aumentando los costos.

Los sistemas muy grandes sólo suelen construirse para fines muy específicos (científicos, industriales, etc.), y en general, su costo de construcción y mantenimiento es muy elevado. Para fijar ideas, cabe mencionar que los sistemas usados en las plantas colectoras de energía solar tienen diámetros típicos entre 5 y $15 \mathrm{~m}$, y las cocinas solares que se comercializan para uso doméstico, entre 1,4 y $1,8 \mathrm{~m}$.

Este último intervalo nos parece apropiado para el proyecto de un aficionado; en tanto que para fines didácticos, entre 0,5 y $1 \mathrm{~m}$ nos parece más que suficiente.

\section{Materiales Apropiados para Construir Reflectores}

\subsection{Generalidades}

Un reflector, como su nombre lo indica, debe tener una alta reflectancia y bajas absorbancia y transmitancia. Además, para asegurar la convergencia de los rayos reflejados, su forma debe permanecer estable ante los esfuerzos internos del ensamblado de sus pétalos, también ante los esfuerzos externos que ocasionalmente podría sufrir, y sobre todo, ante la radiación solar y el aumento de temperatura que ésta provoque. Si bien existen materiales que pueden cumplir todos estos requisitos, en general, no se consiguen fácilmente ni a bajo costo, y en muchos casos, son difíciles de trabajar. Por ello cabe distinguir entre los materiales de "base", los cuales formarán la estructura del reflector y deberán ofrecer las adecuadas propiedades mecánicas y térmicas, y los materiales de "revestimiento" que, aplicados sobre la superficie del sistema, ofrezcan una buena reflectancia.

\subsection{Materiales de base}

Como se adelantó en las secciones anteriores, las propiedades mecánicas de los materiales dependerán del tipo de reflector que se desee construir. Los reflectores paraboloides de diseño convencional admiten materiales de

8 La dispersión de los rayos reflejados aumenta con la disminución de la distancia focal en ambos tipos de modelos, paraboloide y catenoide, pero en el catenoide el efecto es más acentuado. base relativamente más rígidos, y éstos evitan que se deformen; en tanto que el diseño optimizado requiere de materiales relativamente más flexibles (v. §2.4.2). Por su parte, el catenoide debe construirse con materiales muy flexibles, pues debe adoptar su forma por efecto de su propio peso. Pero como ya se discutió respecto de la catenaria (v. §3.1), la condición de flexibilidad no es tan restrictiva y depende fuertemente del tamaño del sistema.

Una buena opción de material de base para construir paraboloides es el acetato de celulosa (especialmente si es blanco reflectivo). Los poliésteres, aunque tienen cierto efecto difusivo, también pueden usarse si son de color blanco brillante y el revestimiento no es demasiado traslúcido. Para el diseño convencional, nos parecen recomendables láminas de poliéster o acetato de espesores del orden de $0,35 \mathrm{~mm}$, y para el diseño optimizado, de $0,15 \mathrm{~mm}$.

Para los catenoides, dependiendo de su tamaño, también se puede usar estos materiales en láminas muy delgadas, telas con componentes sintéticos de acabado brillante, vinilos, o cualquier tipo de material plástico muy flexible y termoestable.

\subsection{Materiales de revestimiento}

Existen diferentes materiales aptos para el revestimiento de reflectores. Muchos de ellos se comercializan en láminas autoadhesivas de aplicación directa o mediante calor, y la mayoría tiene buenas propiedades térmicas y tenacidad, aun en espesores muy delgados. Además de las películas de aluminio anodizado, que tiene muy buena reflectancia, existen opciones más baratas y fáciles de conseguir, como las películas de protección solar para ventanas y las de polarizado de cristales de automóviles, pero son demasiado transparentes y por ello deben ser aplicadas sobre otra superficie blanca brillante que no sea traslúcida.

Algunos tereftalatos de polietileno (conocidos vulgarmente por su sigla en inglés: PET, o por uno de sus nombres comerciales: "Mylar") suelen comercializarse en tiendas de jardinería, para cultivos de interior. Otra opción muy barata y efectiva es el polipropileno biorientado (conocido por su sigla en inglés, BOPP), que suele usarse para fabricar mantas de emergencia y envoltorios de alimentos u otros artículos de uso doméstico. Éste es bastante reflectante y poco transparente, pero su espesor es muy delgado, y debe aplicarse en parches pequeños para obtener una terminación prolija y pareja.

Los vinilos, por su parte, suelen tener la ventaja de ser poco transparentes. Sus espesores típicos son mayores que los del BOPP y el PET, lo cual permite aplicarlos más fácilmente, pero son más caros.

Es conveniente usar materiales "espejados", porque el pico más pronunciado del espectro de radiación solar corresponde a un intervalo de longitudes de onda $(0,1-10) \mu \mathrm{m}$ (ultravioleta cercano, todo el espectro 
visible y parte del infrarrojo) [14]. En este sentido, no son recomendables los materiales de acabado "mate", rugosos, "texturados", ni ninguna otra superficie que provoque reflexión difusa. Los papeles metalizados de uso escolar, por ejemplo, en general no son una buena opción. Tampoco lo es el papel de aluminio de uso culinario, por su fragilidad, ni las pinturas que imitan cromado, porque su reflectancia es insuficiente. Estas últimas acusan otros defectos: en general son caras, difíciles de aplicar en forma pareja, y suelen generar vapores tóxicos.

\subsection{Otros materiales}

Otros materiales muy importantes para obtener un buen resultado final son los pegamentos y los elementos mecánicos utilizados en las junturas de los pétalos. Estos últimos, dependiendo del tamaño y peso del reflector, y de la durabilidad que se desee, pueden ser muy variados: cintas adhesivas, precintas plásticas, cordeles, broches, remaches, etc. Es importante que no deformen la superficie, no alteren su reflectancia, ni provoquen pérdidas de energía.

La elección de los pegamentos, por su parte, suele ser una cuestión crítica. Nos parece muy recomendable usar láminas reflectantes autoadhesivas, porque su aplicación es más sencilla y permiten una terminación prolija y uniforme, minimizando la cantidad de arrugas, burbujas y ondulaciones. En caso de usar películas muy delgadas, un método conveniente para pegarlas sobre el material de base es humedecerlo a éste con detergente muy diluido en agua, y aplicar la lámina reflectante con espátula de goma o plástico, evitando rayaduras. Las láminas reflectantes que no son autoadhesivas pueden aplicarse con el mismo método, usando pegamentos apropiados para materiales plásticos, en cantidades pequeñas, y previamente diluidos, si son demasiado viscosos.

Para pegar, al material de base, el pliego de papel con el diagrama de pétalos, conviene usar pegamento en aerosol o en barra, en cantidades mínimas. Una vez recortado el material de base es recomendable despegar el papel para evitar que éste lo deforme al encogerse en el proceso de secado.

Desde luego, la resistencia a la radiación solar y a las grandes variaciones térmicas son condiciones imprescindibles en todos los materiales.

\section{Modelos Construidos}

Los modelos presentados en las siguientes figuras fueron construidos con fines didácticos.

En la elección de los materiales, primaron las propiedades mecánicas, para los materiales de base, y las propiedades ópticas, para los materiales de revestimiento. Para ambos tipos de materiales (base y revestimiento) también se tuvo en cuenta el bajo costo y la facilidad de trabajo, habida cuenta de la relevancia de estos aspectos en un contexto didáctico típico, en particular, si la construcción de los reflectores se plantea como un proyecto experimental a ser desarrollado por los estudiantes. En este sentido cabe destacar que, de acuerdo a nuestros resultados, con materiales y métodos muy accesibles pueden obtenerse productos que cumplen muy satisfactoriamente su función didáctica.

Para los tres modelos presentados se midieron la reflectancia difusa, $R$, y la transmitancia, $T$, en el rango de longitudes de onda desde 380 hasta $1068 \mathrm{~nm}$, se calcularon promedios, y se obtuvo la absorbancia promedio, $\bar{A}$, por diferencia: $\bar{R}+\bar{T}+\bar{A}=1$. La reflectancia se midió con un Espectrorradiómetro PhotoResearch PR-715, y la transmitancia, con un Espectrorradiómetro Optronic OL 750 .

La Fig. 13 muestra dos fotografías de un reflector paraboloide de diseño convencional, de 0,47 m de diámetro (área efectiva: $\sim 0,1735 \mathrm{~m}^{2}$ ), construido con una lámina de acetato revestida de tereftalato reflectante. En el panel superior $(a)$, se observa que los rayos convergen sobre el eje del paraboloide en un rango de $\sim 0,07 \mathrm{~m}$ (recuadro rojo); y en el panel inferior (b), se aprecia el área de convergencia a la altura del foco (delimitada con una circunferencia roja), la cual mide $\sim 0,005 \mathrm{~m}^{2}$. En este modelo, $\bar{R} \cong 0,81, \bar{T} \cong 0,01$ y $\bar{A} \cong 0,18$.

La Fig. 14 muestra dos fotografías de un reflector paraboloide de diseño optimizado, construido con una lámina cuadrada de poliéster, de $0,345 \mathrm{~m}$ de lado, revestida de tereftalato reflectante. En el panel de la derecha (b), se observa que los rayos reflejados en el paraboloide se concentran en la palma de la mano de la persona que lo sostiene. En este modelo, $\bar{R} \cong 0,58, \bar{T} \cong 0,05 \mathrm{y}$ $\bar{A} \cong 0,37$.

La Fig. 15 presenta dos fotografías de un reflector catenoide de diseño sencillo, de ocho pétalos de $0,4 \mathrm{~m}$ de

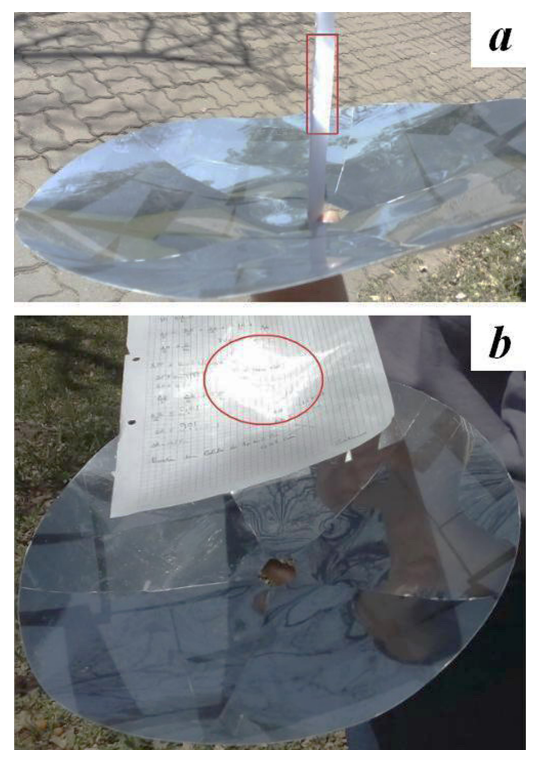

Figura 13: Paraboloide de diseño convencional. En el panel superior (a) se aprecia el rango de convergencia en altura, y en el inferior (b), el área de convergencia, a la altura del foco. 


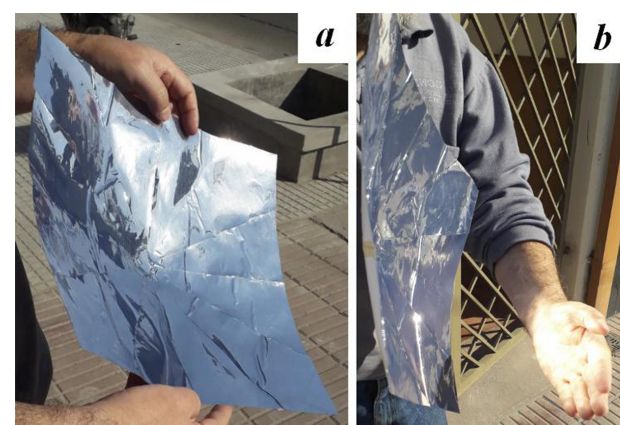

Figura 14: Paraboloide de diseño optimizado. a) Vista general. b) Convergencia de los rayos reflejados.

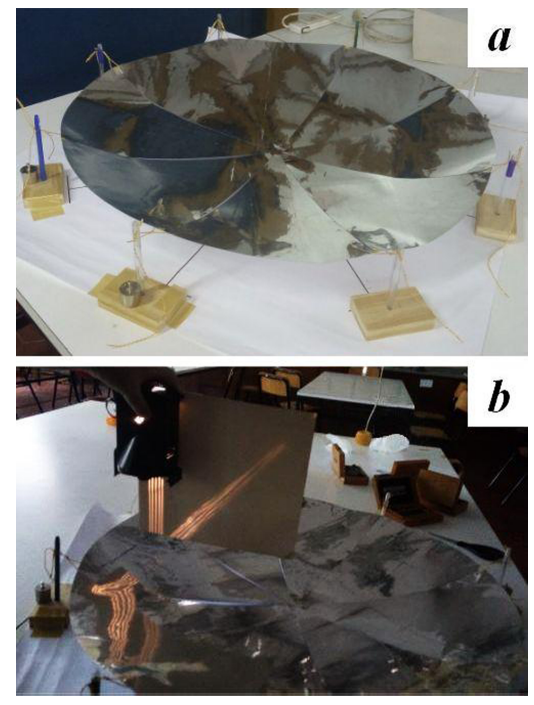

Figura 15: Catenoide de diseño sencillo. a) Vista general. b) Convergencia de los rayos reflejados.

longitud, construido con una lámina delgada de poliéster revestida de vinilo reflectante. En el panel superior $(a)$ se observa una vista general del reflector, suspendido mediante ocho hilos, cada uno de los cuales tiene un extremo adherido a un punto de suspensión (puntos azules de la Fig. 10). El extremo opuesto de cada hilo está enhebrado en un soporte, desde donde su longitud puede ajustarse hasta que todos los puntos de suspensión quedan a una misma altura $z_{s}$ (v. §3.3.1). En el panel inferior $(b)$, se observan cinco haces de rayos paralelos que inciden verticalmente y se reflejan convergiendo hacia un mismo punto, situado en el eje del catenoide. En este modelo, $\bar{R} \cong 0,64, \bar{T} \cong 0,06$ y $\bar{A} \cong 0,30$.

\section{Conclusiones}

Los reflectores solares constituyen un recurso de una gran riqueza didáctica y ofrecen un valor formativo en cuanto concierne al uso responsable de energías y recursos. Además, tienen utilidad práctica, y su construcción y uso es muy motivador para los estudiantes.
Se los puede construir fácilmente, con materiales accesibles y baratos. Los acetatos, poliésteres y otros materiales plásticos flexibles y termoestables son recomendables, en general, como materiales de base, en tanto que los polietilenos y el vinilo son una buena opción como reflectantes. La elección de estos materiales dependerá del modelo, el diseño y, sobre todo, la finalidad del sistema. Esta última debe ser, también, la razón fundamental para decidir sus dimensiones.

Para diseñar los reflectores de manera eficiente, se ha desarrollado un análisis geométrico que permite obtener resultados precisos sobre diversas cuestiones puntuales tales como el área efectiva, la distancia focal y el número de pétalos. Esto permitirá al profesional o estudiante avanzado desarrollar un sentido crítico para elegir el diseño más adecuado a sus fines, sopesar las exigencias de la construcción en términos de la eficacia del producto final, y fundamentar decisiones sobre los materiales, su aprovechamiento, el dimensionamiento de los parámetros, etc. Toda esta labor se ha facilitado y sistematizado mediante la provisión de programas de nuestra autoría, desarrollados en Scilab 5.5.1, los cuales resuelven las principales cuestiones críticas del diseño.

En este trabajo se ha presentado un nuevo modelo, el catenoide, que por su naturaleza puede construirse con materiales muy flexibles y livianos. Esto facilita su construcción, manipulación y mantenimiento, todo lo cual lo hace idóneo para sistemas de gran tamaño. Su principal desventaja radica en la imposibilidad de orientarlo hacia el sol, pero esto se puede soslayar, en gran medida, con un dimensionamiento adecuado de la distancia focal, y usando el recurso de desplazar el objeto a irradiar hacia la posición más conveniente.

A modo de ejemplo e ilustración de los modelos desarrollados, se presentan tres sistemas construidos con fines didácticos, que muestran una buena convergencia de los rayos reflejados. En un próximo trabajo, los autores proyectan construir sistemas de mayores dimensiones para evaluar, en aplicaciones prácticas tales como cocinas solares, la eficiencia de estos diseños y la pertinencia del uso de materiales de bajo costo.

\section{Reconocimientos}

Este trabajo se realizó en el marco del proyecto "Investigación Científica para el Perfeccionamiento Docente en Física" (Cód. E629), subvencionado por la Universidad Nacional de Tucumán.

Agradecemos al Mg. Ing. Sergio Gor y a la Ing. Bárbara Silva, del Dpto. de Luminotecnia de la UNT, por su valiosa y generosa colaboración en la medición de las propiedades ópticas de los materiales empleados en los modelos construidos. 


\section{Referencias}

[1] P. Melogno, P. Rodríguez y S. Fernández, Elementos de Historia de la Ciencia (Unidad de Comunicación de la Universidad de la República, Montevideo, 2011).

[2] T.L. Heath, The Thirteen Books of Euclid's Elements (Cambridge University Press, London, 1908).

[3] S.C. Tucker, Battles that Changed History (ABC-Clio, Santa Barbara, 2010), p. 54.

[4] A. Riahi, A. Ben Haj Ali, A.A. Guizani y M.J. Balghouthi, Mater. Environ. Sci. 9, 8 (2018).

[5] J. Aidan, IOSR Journal of Applied Physics 6, 5 (2014).

[6] I.L. Mohamed, International Journal of Engineering Research and Applications 3, 4 (2013).

[7] D.Y. Dasin, A.A. Asere y D. Habou, Nigerian Journal of Solar Energy 22, 52 (2011).

[8] S. Adil Ahmed, N.S. Prasanna Rao, P.L. Srinivas Murthy y B.P. Terani, International Research Journal of Engineering and Technology 2, 4 (2015).

[9] J. Manukaji, IOSR Journal of Engineering 5, 1 (2015).

[10] M.A. Torres Rodrigues y L.F. Mackedanz, Rev. Bras. Ensino Fís. 40, 1 (2018).

[11] N. Piskunov, Cálculo Diferencial e Integral (Editorial Limusa, Ciudad de México, 2006).

[12] https://drive.google.com/open?id= 1kgIiFI5LFiX88qmR61y5f xnKjPf JhFd0

[13] G. Woan, The Cambridge Handbook of Physics Formulas (Cambridge University Press, Cambridge, 2000).

[14] S. Akasofu y S. Chapman, Solar Terrestrial Physics (Oxford University Press, London, 1972). 